\title{
Impact on patients' health status following early identification of a COPD exacerbation
}

\author{
J. Bourbeau*, G. Ford” ${ }^{\#}$ H. Zackon`, N. Pinsky+, J. Lee ${ }^{\S}$ and G. Ruberto ${ }^{\S}$
}

ABSTRACT: The current study aimed to assess the impact on patient health status during an acute exacerbation of chronic obstructive pulmonary disease (AECOPD).

A total of 421 COPD patients were enrolled in a multicentre, single-arm study with a 6-month observational follow-up period. Patients received two inhalations of Symbicort 200 Turbuhaler ${ }^{\circledR}$ twice a day. Patients were assessed before the run-in period, at baseline and at 1, 3 and 6 months. Patients were instructed to report a change in respiratory symptoms lasting $>\mathbf{2 4} \mathbf{~ h}$. This defined an AECOPD. In addition to the initial call, the St George's Respiratory Questionnaire (SGRQ), COPD Control Questionnaire (CCQ), Medical Research Council (MRC) dyspnoea scale and activities of daily living (ADL) were completed at 5-7 and 12-14 days.

A group of 176 patients reported at least one AECOPD. Exacerbations were associated with statistically significant mean changes (worsening) in the SGRQ activity and impact domains at onset (mean \pm SD $12.1 \pm 18.1$ and $14.0 \pm 15.2)$, during the first $(9.8 \pm 19.0$ and $9.4 \pm 16.6$ ) and second weeks $(3.1 \pm 15.5$ and $3.3 \pm 14.7)$. Clinically significant deterioration in SGRQ impact scores was shown in $71 \%$ of patients following early identification, with 55 and $37 \%$ during the first and second weeks of an AECOPD, respectively.

Acute exacerbation severely impacts on health status. The current study provides valuable information on the change in health status during an acute exacerbation of chronic obstructive pulmonary disease that can be utilised for future trials that evaluate therapeutic intervention.

KEYWORDS: Chronic obstructive pulmonary disease, exacerbation, health-related quality of life

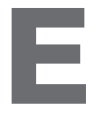
xacerbations are common for many patients with chronic obstructive pulmonary disease (COPD) and contribute greatly to an increase in morbidity, frequent emergency department (ED) visits, hospital admissions, and increased healthcare costs [1-3]. Evidence suggests that patients with recurrent acute exacerbations of COPD (AECOPD) have faster decline in lung function $[4,5]$, possibly due to increased rate of airway inflammation [6]. The impact of AECOPD on patients' health status is far from negligible [7-9].

MiravitLles et al. [7] assessed the long-term evolution of COPD patients' health status followed prospectively over a 2-yr period. They showed that patients with frequent exacerbations had 2 units $\cdot \mathrm{yr}^{-1}$ worsening of the St George's Respiratory Questionnaire (SGRQ) total score, compared with those with infrequent exacerbations. Similarly, hospital admissions resulted in an increased change of almost 2 units $\cdot \mathrm{yr}^{-1}$. In a clinical trial, SPENCER and JONES [9] assessed the rate of recovery following an exacerbation. The greatest improvement in the SGRQ score occurred within the first 4 weeks (mean 8.9
(95\% confidence interval 6.5-11.5) units). A further improvement of 4.1 (2.2-5.9) units, constituting a clinically important improvement, was noted in patients with no recurrence of exacerbation. There is no data on loss of health during early identification of an AECOPD.

In the current study, the authors prospectively followed a cohort of 421 patients with moderateto-severe COPD. These patients had their health status assessed over a 6-month period and following early identification of an AECOPD. The rationale of the trial was to compile appropriate knowledge to be able to develop future studies evaluating therapeutic interventions for a pragmatic control plan. The primary objective was to assess the impact on patients' health status (as measured by SGRQ) following early identification of an AECOPD. The secondary objectives were to assess the impact of an AECOPD on disease control in terms of symptoms, and functional and mental state changes, as measured by the COPD Control Questionnaire (CCQ), Medical Research Council (MRC) dyspnoea scale and activity of daily living (ADL). The rate of improvement (return to baseline) in health status,
AFFILIATIONS

*Respiratory Epidemiology and Clinical Research Unit, Montreal Chest Institute of the Royal Victoria Hospital,

'Dept of Medicine, St Mary's Hospital, McGill University Health Center, Montréal, QC,

\#Dept of Medicine, University of Calgary, Calgary, AB,

${ }^{+}$Family Medicine, Halifax, NS, and ${ }^{\S}$ AstraZeneca Canada Inc. Missisauga, ON, Canada.

CORRESPONDENCE

J. Bourbeau

Respiratory Epidemiology and

Clinical Research Unit

Montreal Chest Institute

3650 St.Urbain

Montréal

QC H2X 2P4

Canada.

Fax: 15148432083

E-mail: jean.bourbeau@mcgill.ca

Received:

December 222006

Accepted after revision:

July 272007

STATEMENT OF INTEREST

A statement of interest for this study

can be found at

www.erj.ersjournals.com/misc/

statements.shtml

European Respiratory Journal

Print ISSN 0903-1936

Online ISSN 1399-3003 
disease control and ADL in patients associated with an AECOPD were measured, and the predictive factors of exacerbation were assessed. The information from the current study will increase understanding of the impact on health status both at onset and during AECOPD. Knowledge of the change in health status of patients with stable and moderateto-severe COPD, at the onset and during an AECOPD, could be utilised in designing and evaluating therapeutic intervention(s) aimed at improving the management of AECOPD in future trials.

\section{METHODS}

\section{Study patients}

Patients were recruited from 59 participating centres across Canada between February 6, 2003 and April 21, 2004, based on the following eligibility criteria: 1 ) diagnosis of stable COPD; 2 ) aged $\geqslant 40$ yrs; 3 ) smoking history $\geqslant 10$ pack-yrs; 4) forced expiratory volume in one second (FEV1) $\leqslant 70 \%$ of predicted value and FEV1/ forced vital capacity (FVC) $<0.70$; 5) dyspnoea $\geqslant 2$ on the MRC scale; at least two exacerbations requiring medical intervention in the previous 3 yrs; 6) no history of asthma or allergic rhinitis before the age of 40;7) no regular use of oxygen, beta blockers, oral corticosteroids or the combination of inhaled corticosteroids/long-acting $\beta_{2}$-agonists; and 8) no unstable or life-threatening comorbid condition. All patients gave informed consent to participate in the study. Additional criteria to be fulfilled at visit 2 were: 1) no AECOPD during the run-in period requiring intervention, unscheduled physician or ED visit or hospitalisation; 2) no course of antibiotic(s) and/or oral corticosteroids; and 3) no increase in inhaled steroid and/or parenteral steroid treatment.

\section{Study design}

The study was a multicentre, single-arm prospective cohort with a 2-week run-in period followed by a 6-month observational period, during which patients received two inhalations of budesonide/formoterol 200/6 $\mu \mathrm{g}$ (Symbicort 200 Turbuhaler ${ }^{\circledR}$; AstraZeneca, Mississauga, ON, Canada) twice daily as maintenance treatment. The run-in period was used to ensure the patients were stable. Patients' assessment included a complete medical history, spirometry, health status, disease control, the modified MRC and activity of daily living. Health status and disease control were measured prior to the 2-week run-in period, after the run-in period, just before the treatment period (baseline), and at 1, 3 and 6 months. All patients were asked to record daily, in a diary, their symptoms (dyspnoea, cough, sputum quantity and colour, sleep disturbances), morning peak expiratory flow and use of rescue medication. Patients were reminded at monthly intervals to complete diary records and also had follow-up telephone interviews after they reported acute exacerbations.

\section{Reporting exacerbations and early identification}

Patients were asked to report to their investigative site if they had sustained (for $>24 \mathrm{~h}$ ) worsening of at least one of the three cardinal symptoms of AECOPD: dyspnoea, sputum volume and purulence. This defined a patient who experienced an AECOPD. The first day of change in symptoms was taken as the day of onset of exacerbation. In addition to the initial patient call, follow-up calls were made at 5-7 days and 12-14 days from the day of onset. Each telephone interview included the completion of the SGRQ (excluding the symptoms domain) and a reminder to the subjects to complete the exacerbation notebook (self-administered CCQ, modified MRC and ADL) at home. The research assistant did not provide any intervention but encouraged subjects to follow their usual care plan or other channel(s) for intervention. The research assistant was trained with regards to an action plan in the event of an emergency situation related to an AECOPD.

\section{SGRQ}

The SGRQ is a 50-item questionnaire with 76 weighted responses. It provides a total score and three domain scores: symptoms (respiratory symptoms); activity (physical activities that cause or are limited by breathlessness); and impact (social and psychological effects of the disease). The symptoms domain refers to the last year and the activity and impact domains to current symptoms. The SGRQ is scored from 0-100 where 0 indicates the best and 100 the worst health. The SGRQ is a disease-specific questionnaire developed for patients with chronic airway disease. Its validity and reproducibility have been confirmed $[10,11]$. As the symptoms component of SGRQ cannot be used in frequent administrations ( $<1$ month interval), only the activity and impact components were utilised during the exacerbation telephone calls [12].

\section{$C C Q$}

The CCQ is a self-administered multidimensional symptom control questionnaire that includes 10 questions in three domains: symptoms, functional state and mental state. It has been shown to have strong evaluative and discriminative measurement properties [13]. Patients are asked to recall their experiences during the previous $24 \mathrm{~h}$ and to respond to each of the 10 questions using a 7-point scale from $0=$ asymptomatic/ no limitation to $6=$ extremely symptomatic/totally limited.

\section{$A D L$}

The ADL questionnaire used in the current study is not standardised and has not been validated. Subjects were asked "How much did your COPD affect your normal daily activities during the previous $24 \mathrm{~h}$ ?" and were required to indicate, by placing a cross on a $10-\mathrm{cm}$ scale (with divisions ranging from 0-100\%), how their COPD affected their normal daily activities. For example: 1) if activities were not affected at all by COPD, the cross would be placed at the $0 \%$ mark; 2) if activities were reduced by half, the cross would be placed at the $50 \%$ mark; and 3) if unable to perform any of their daily activities, the cross would be placed at the $100 \%$ mark.

\section{MRC dyspnoea scale}

The MRC dyspnoea scale is a commonly used, validated, ordinal scale on which patients rate the type and magnitude of their dyspnoea according to five grades of increasing severity [14]. The MRC dyspnoea scale was interviewer-administered at scheduled clinic visits from visit 2 onwards and with each telephone call related to COPD exacerbations.

\section{EuroQol-5D}

The EuroQol (EQ)-5D is a multidimensional measure of healthrelated quality of life. EQ-5D consists of the EQ-5D descriptive system (a page for obtaining a self-reported description of health status) and EQ-5D visual analogue scale (VAS), a 
"thermometer" for eliciting a self-rating of health status [15]. The EQ-5D was administered at each clinic visit with visit 1 as a practice session for the completion of the questionnaire. The EQ-5D data at visit 1 was not entered into the database. Visit 2 responses served as baseline values. In addition to the completion of the EQ-5D at each scheduled clinic visit, the questionnaire was also completed at the time of each telephone call related to an exacerbation of COPD.

\section{Statistical analysis}

Patients who had completed the 2-week run-in period to ensure their disease was stable and received at least one dose of study medication, were considered for the analysis. Results are expressed as mean $\pm \mathrm{SD}$, median (interquartile range) where appropriate. A p-value of $<0.05$ was considered statistically significant.

For subjects experiencing COPD exacerbations, all efficacy variables including the questionnaires SGRQ, CCQ, MRC, EQ$5 \mathrm{D}$, and ADL were presented over the four phases of the first exacerbation. Only data within \pm 1 day deviation were included in the final analysis (i.e. baseline, 0-3 days for the onset, 4-8 days for 5-7 days post-exacerbation and 11-15 days for 12-14 days post exacerbation). Baseline health-related quality of life was determined by the most recent SGRQ score taken $\geqslant 48 \mathrm{~h}$ prior to early identification of an exacerbation. Changes from baseline were analysed using paired t-tests. Correlations and effect sizes were calculated to compare changes between questionnaires.

Linear extrapolation using the last two data points (5-7 days and 12-14 days) was used to estimate the time that it takes in health status, disease control and ADL to return to baseline after the first exacerbation. To assess the predicting factors on exacerbation, an analysis was performed using a logistic regression model of having one or more AECOPD versus no AECOPD. Potential predictors included in the logistic analysis were age, sex, education level, smoking history, years since diagnosis, lung function, dyspnoea (MRC scale), number of exacerbations in the previous 3 yrs and number of months since the last event, nebulised bronchodilators, antibiotic and oral corticosteroids required for the last exacerbation, hospitalisation in the last year and ever taking part in pulmonary rehabilitation. Using a backward eliminating technique, statistically significant variables were kept in the model when $\mathrm{p}<0.10$. Age had to be included in the final model.

\section{RESULTS}

\section{Patients}

Baseline characteristics of all 421 patients, 245 patients without exacerbation, 135 with one, and 41 with two or more exacerbations are summarised in table 1. Patient characteristics were similar except among patients who experienced two or more AECOPD. These patients had more AECOPD in the past, increased use of short-acting $\beta_{2}$-agonists, increased baseline dyspnoea, worse health status and were more likely to have taken part in pulmonary rehabilitation.

\section{Reported exacerbations}

During the 6-month observational period, 176 (41.8\%) patients reported a deterioration of at least one cardinal respiratory symptom, 135 patients had a single exacerbation and 41 had multiple exacerbations (28 subjects had two exacerbations, 10 subjects had three exacerbations and three subjects had four exacerbations). At the onset of AECOPD, increased cough and dyspnoea worsening were equally reported by patients ( 69 and $65 \%$, respectively). Most patients (63\%) presented with additional symptoms to change in dyspnoea (i.e. change in sputum colour and/or amount, wheezing and/or chest tightness, coughing, colds and sore throat). Specifically, 61\% of patients reporting exacerbations experienced increased amounts of sputum and $41 \%$ reported change in sputum colour.

\section{Health status}

Health status improved after the first month of treatment and remained stable for the duration of the study for patients who had no AECOPD (SGRQ total score mean change of -6.2 at 1 month and -6.9 at 6 months) and those with only one exacerbation (SGRQ total score mean change of -4.7 at 1 month and -4.2 at 6 months; fig. 1). Patients with two or more exacerbations did not improve after the first month but their health status remained unchanged during the 6-month study period.

Table 2 shows baseline health status and changes following early identification of an AECOPD. AECOPD are associated with statistically significant mean changes in the SGRQ activity and impact domains at onset, during the first and second weeks. Fig. 2 shows the percentage of patients with a clinically important SGRQ impact score worsening (increase of $\geqslant 4$ from baseline). Clinically significant deterioration in SGRQ impact scores was demonstrated in $71 \%$ of patients during onset of AECOPD, 55\% during the first week and 37\% during the second week.

\section{Other clinical outcomes}

Table 3 shows changes in patient responses following early identification of COPD exacerbations. Generally, patients with an AECOPD showed statistically and clinically significant worsening of their disease. This was indicated by patients' responses to the CCQ, the three domains (symptom, functional and emotional state), the MRC dyspnoea scale, and the ADL questionnaires. The correlations between changes from the SGRQ and the other clinical outcomes were strong between variables (i.e. $\geqslant 0.5$ ) and remained stable over time (i.e. no significant changes in correlations from baseline to 14 days after onset; data not shown). For the change from baseline to onset of exacerbation, the effect sizes were within a moderate and high range $(0.6-0.9)$ for most outcome variables, with the exception of ADL (0.3). The impact domain appears to be more responsive to acute exacerbation as it showed a larger change compared with the activity domain, based on the effect size (data not shown).

\section{Rate of improvement}

The estimated time for health status (SGRQ) to return to baseline was 11 and 9 days for the impact and activity domain, respectively. Symptoms and functional state, as measured by the CCQ, returned to baseline at 12 and 13 days, respectively, while mental state took much longer (39 days). The time for patients to return to their activities of daily living was 18 days. 


\begin{tabular}{|c|c|c|c|c|}
\hline Characteristic & All patients & No exacerbation & 1 exacerbation & $\geqslant 2$ exacerbations \\
\hline Subjects & 421 & 245 & 135 & 41 \\
\hline Male & $239(57)$ & $142(58)$ & $79(59)$ & $18(44)$ \\
\hline Age & $66(41-88)$ & $67(41-88)$ & $66(45-86)$ & $64(44-80)$ \\
\hline Pre-BD FEV $1 \%$ pred $\leqslant \mathbf{5 0} \%$ & $275(65)$ & $158(64)$ & $87(64)$ & $30(73)$ \\
\hline FEV1/FVC pre-BD & $0.52(0.24-0.88)$ & $0.52(0.24-0.80)$ & $0.51(0.26-0.71)$ & $0.50(0.29-0.88)$ \\
\hline Current smoker & $168(40)$ & $94(38)$ & $60(44)$ & $14(34)$ \\
\hline Smoking habits pack-yrs & $46(10-150)$ & $47(11-150)$ & $45(10-117)$ & $41(12-98)$ \\
\hline Years since COPD diagnosis ${ }^{\#}$ & $8(0.0-60.0)$ & $8(0-60.0)$ & $8(0.1-28.1)$ & $9(0.2-32.8)$ \\
\hline \multicolumn{5}{|l|}{ Exacerbations } \\
\hline Anticholinergic & $154(37)$ & $79(32)$ & $55(41)$ & $20(49)$ \\
\hline Combined SABA and anticholinergic & $134(32)$ & $78(32)$ & $44(33)$ & $12(29)$ \\
\hline ICS & $209(50)$ & $121(49)$ & $67(50)$ & $21(51)$ \\
\hline LABA & $61(14)$ & $32(13)$ & $22(16)$ & $7(17)$ \\
\hline Combined LABA and ICS & $4(1)$ & $2(0.8)$ & $2(1.5)$ & $0(0)$ \\
\hline $\begin{array}{l}\text { Participation in pulmonary rehabilitation } \\
\text { programme }\end{array}$ & $28(6.7)$ & $8(3.3)$ & $13(9.6)$ & $7(17.1)$ \\
\hline MRC dyspnoea & $2.9(2-5)$ & $2.8(2-5)$ & $2.9(2-4)$ & $3.2(2-4)$ \\
\hline \multicolumn{5}{|l|}{ SGRQ } \\
\hline Activity score & $62.9(0-100)$ & $62.1(0-100)$ & $63.1(0-100)$ & $67.7(35.6-92.9)$ \\
\hline Impact score & $31.8(0-83.8)$ & $30.9(0-83.8)$ & $32.8(0-83.6)$ & $33.9(1.6-65.3)$ \\
\hline
\end{tabular}

Data are presented as $n, n$ (\%) or mean (range). FEV1: forced expiratory volume in one second; \% pred: \% predicted; BD: bronchodilator; FVC: forced vital capacity; SABA: short-acting $\beta_{2}$-agonist; ICS: inhaled corticosteroids; LABA: long-acting $\beta_{2}$-agonists; MRC: Medical Research Council; SGRQ: St George's Respiratory Questionnaire. ${ }^{\#}$ : there were two subjects with a diagnosis of COPD occurring at visit 1 (yr since COPD diagnosis: 0). All patients were started on combination ICS/LABA as part of standard therapy in the study.

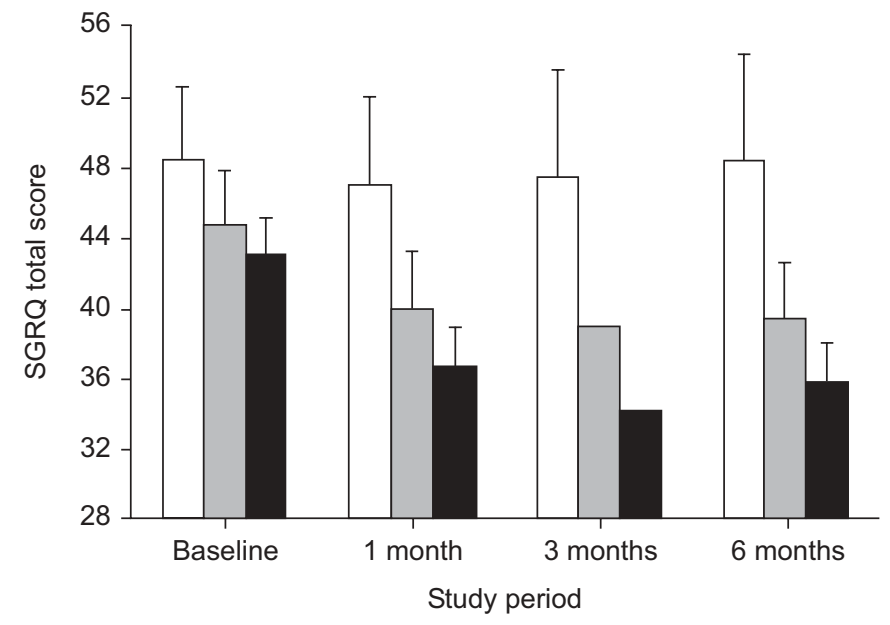

FIGURE 1. Total St George's Respiratory Questionnaire (SGRQ) score according to patient's exacerbation frequency during the study period. Data are

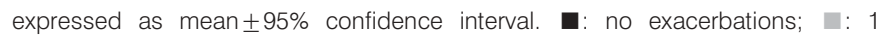
exacerbation; $\square: \geqslant 2$ exacerbations.

\section{Predicting factors of exacerbation}

The results of the regression model analysis are presented in table 4. Predictive factors of exacerbation included age, dyspnoea (MRC scale) and the number of exacerbations in the past year. It also appeared that those who participated in a pulmonary rehabilitation programme had higher odds of exacerbation compared with those who did not participate.

\section{DISCUSSION}

The current study revealed a clinically significant deterioration in health status during an AECOPD. This was reflected as a high magnitude on the SGRQ impact domain (increase of $\geqslant 4$ ), which persisted in more than half of patients during the first week and one-third of patients during the second week. Based on the linear regression extrapolation, most of the variables of health status, symptoms and functional state returned to baseline after 14 days with the exception of mental state (39 days). AECOPD clearly impacts not only on the functional but also on the emotional state of patients, and markedly restricts activities of daily living.

The present study also showed that increased dyspnoea and previous exacerbations are predictors of exacerbations. Those 


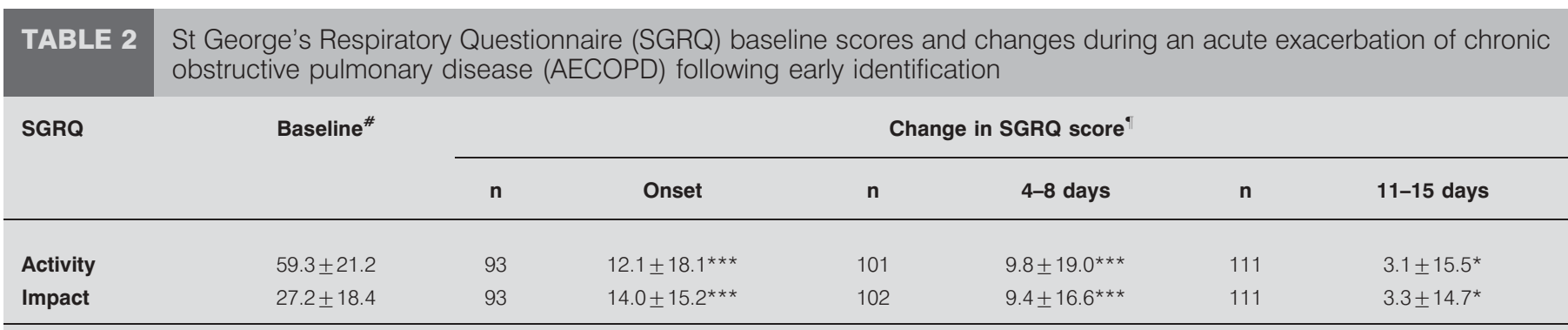

Data are presented as mean $\pm \mathrm{SD}$, unless otherwise stated. *: the most recent SGRQ score taken $\geqslant 48 \mathrm{~h}$ prior to an AECOPD (mean \pm SD time delay between baseline and exacerbation was $33.2 \pm 22.3$ days); $"$ : change from the most recent score taken $\geqslant 48 \mathrm{~h}$ prior to an AECOPD. *: $p<0.05 ;{ }^{* * *}: \mathrm{p}<0.001$.

who participated in a pulmonary rehabilitation programme had higher odds of an AECOPD compared with those who did not participate. This is likely to represent a bias from confounding rather than the effect of the exposure to a pulmonary rehabilitation programme itself.

The pathophysiology of AECOPD is poorly understood and it is often difficult to distinguish true exacerbations from normal day-to-day variations of COPD. In 2000, a consensus panel of respiratory physicians from Europe and the USA suggested that an exacerbation of COPD should be defined as "a sustained worsening of the patient's condition, from stable state and beyond normal day-to-day variations, that is acute in onset and necessitates a change in regular medication in a patient with underlying COPD" [13]. Subsequently, the definition was amended to include exacerbations that did not necessitate a change in treatment [4]. In the current study, a symptom-based definition was used to ensure that the main focus would be on patients' perceptions of their symptoms.

It is well known that AECOPD have a considerable impact on patients with respect to disease progression [4, 16], morbidity [16], mortality and health status [7-9, 17]. In patients admitted to intensive care units for AECOPD, it was shown that after 6 months only $26 \%$ were alive and able to report a good-toexcellent health status [3]. In the East London Cohort studies [17], 35 days after the onset of an AECOPD, PEF and symptoms scores had not returned to baseline levels in 24.8

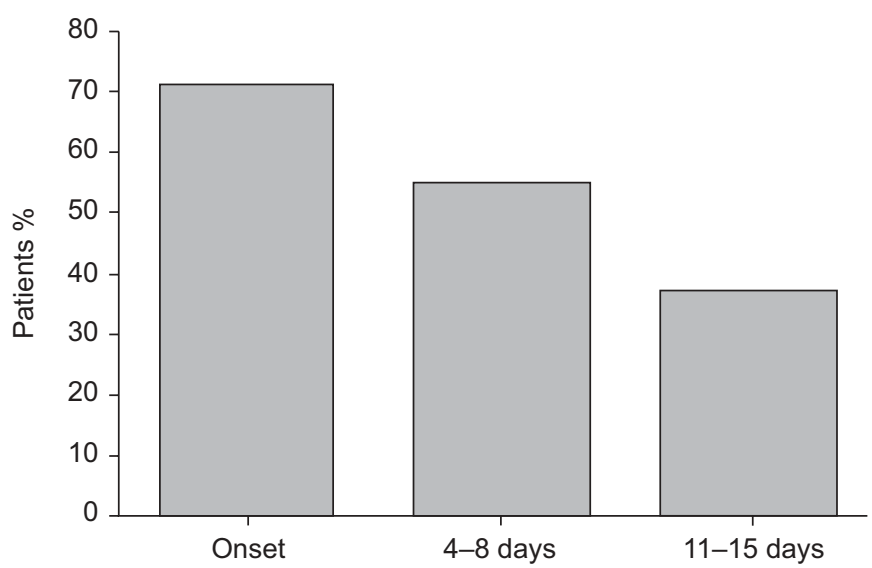

FIGURE 2. Percentage of patients with St George's Respiratory Questionnaire impact score worsening (scores $\geqslant 4$ from baseline) and $13.9 \%$ of patients, respectivley. In another study by SPENCER and JONES [9], the rapid improvement observed during the first 4 weeks was followed by a slower recovery of up to 6 months in some patients. Their study utilised a methodology different to the current study, which makes comparison difficult. SPENCER and JONES [9] recruited patients with an acute exacerbation and did not assess patients while they were stable. However, improvement over the first 4 weeks was greater than over the subsequent 5 months. This seems to be in general agreement with the present study results.

The current study is the first to report on health status and worsening symptoms immediately following the identification of an AECOPD. The study indicates that from the onset of an AECOPD, and for up to 2 weeks, most patients are disabled to the point of being limited in their activities of daily living and experience a noticeably reduced quality of life. These results suggest that the timing and choice of treatment of an AECOPD may be based on early symptom changes, loss of disease control (symptom, functional or mental state) and impact on health status. There was large variability in changes in the SGRQ scores. This is not unexpected based on what clinicians see in their clinical practice. The current study also presented deterioration with respect to the percentage of patients with clinically important deterioration (SGRQ increase $\geqslant 4$ ), which may be more meaningful.

The main strengths of the present study are: that COPD patients were enrolled while their disease was stable, thus ensuring knowledge of their baseline symptoms and health status; that they were followed prospectively; and that patientcentred outcomes were assessed at the onset of an AECOPD and during their short-term recovery. The present cohort is likely to be representative of the COPD population known to be at risk for AECOPD considering the large sampling across the country (59 participating centres) and the participation of primary care as well as hospital-based clinics. The definition of exacerbation used in the study was not too restrictive while at the same time conforming to the consensus definition. While exclusion of a single symptom event could have reduced the false positives arising from natural variability of the disease, it could also have excluded true mild exacerbations. However, study results may apply only to patients who report exacerbations. This may be an important element in healthcare definitions of exacerbations that is often overlooked. Some patients may not report frequent day-to-day symptom changes because they become accustomed to them. For example, 


\begin{tabular}{|c|c|c|c|c|c|c|c|}
\hline \multirow[t]{2}{*}{ Questionnaire } & \multirow[t]{2}{*}{ Baseline } & \multicolumn{6}{|c|}{ Change from baseline } \\
\hline & & $\mathbf{n}$ & Onset & $\mathbf{n}$ & 4-8 days & $\mathbf{n}$ & 11-15 days \\
\hline \multicolumn{8}{|l|}{$\mathrm{CCQ}$} \\
\hline Symptoms & $2.4 \pm 1.2$ & 89 & $1.0 \pm 1.3^{\star \star \star}$ & 97 & $0.8 \pm 1.4^{\star \star \star}$ & 101 & $0.04 \pm 1.2$ \\
\hline Functional state & $2.1 \pm 1.3$ & 90 & $0.5 \pm 1.3^{\star \star \star}$ & 97 & $0.6 \pm 1.5^{\star \star \star}$ & 101 & $0.2 \pm 1.2$ \\
\hline Mental state & $2.0 \pm 1.8$ & 85 & $0.8 \pm 1.7^{\star \star *}$ & 95 & $0.6 \pm 1.6^{\star \star \star}$ & 98 & $0.2 \pm 1.6$ \\
\hline Total score & $2.2 \pm 1.2$ & 84 & $0.8 \pm 1.1^{\star \star \star}$ & 95 & $0.7 \pm 1.2^{\star \star \star}$ & 98 & $0.1 \pm 1.1$ \\
\hline EQ-5D health score & $66.6 \pm 17.8$ & 91 & $-12.6 \pm 19.0^{*}$ & 96 & $-9.0 \pm 18.2^{\star}$ & 101 & $-1.6 \pm 16.2$ \\
\hline MRC dyspnoea score & $3.0 \pm 0.8$ & 97 & $0.5 \pm 0.8^{\star \star \star}$ & 104 & $0.3 \pm 0.8^{\star \star \star}$ & 116 & $0.0 \pm 0.9$ \\
\hline ADL score & $32.8 \pm 26.9$ & 91 & $8.7 \pm 31.3^{\circ}$ & 96 & $10.2 \pm 34.2^{\#}$ & 100 & $3.7 \pm 30.1$ \\
\hline
\end{tabular}

another study [17] estimated that patients failed to report up to $50 \%$ of symptom-based exacerbations.

Preventing AECOPD and improving the prognosis should represent a key treatment goal. Guidelines [18, 19] have emphasised the importance of regular therapies, such as long-acting anticholinergics [20], inhaled corticosteroids [21] and combination therapy [22-25] to prevent an AECOPD. Prevention of these exacerbations may help to slow disease progression and impact on health status. Shortening the duration and severity of an AECOPD should also be considered as an important outcome in the management of COPD. Recently, it has been demonstrated that early treatment of an AECOPD can lead to faster recovery from symptoms, improved health status and reduced risk of hospital admission [26]. Other studies also suggest that patients who use selfmanagement action plans to promptly treat exacerbations have $40 \%$ fewer hospital admissions [1, 27]. Health status has become important in the validation of specific treatments used for AECOPD not solely based on the prevention of the AECOPD but also on the time to recovery.

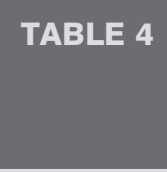

Predictive factors for an acute exacerbation of chronic obstructive pulmonary disease (AECOPD), having at least one versus no exacerbations during the 6-month follow-up

\begin{tabular}{lcc} 
Factor & Adjusted OR (95\% Cl) & p-value \\
\hline Age $^{\#}$ & $0.98(0.96-1.00)$ & 0.077 \\
Dyspnoea $^{*}$ & $1.35(1.06-1.74)$ & 0.017 \\
AECOPD $^{+}$in the past 3 yrs n & $1.14(1.06-1.23)$ & 0.0008 \\
Pulmonary rehabilitation $^{\mathbf{s}}$ & $0.32(0.15-0.66)$ & 0.0021 \\
\hline
\end{tabular}

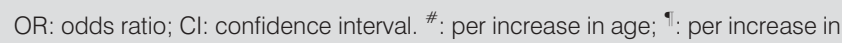
Medical Research Council dyspnoea scale; ${ }^{+}$: per increase in exacerbation; s: difference for patients who did not take part in a rehabilitation programme compared with those who did.
In summary, the present study has demonstrated that health status can be effectively measured during an acute exacerbation of chronic obstructive pulmonary disease. Acute exacerbations of chronic obstructive pulmonary disease are severely distressing events that impact greatly on health status, loss of symptom control and functional state, and have a prolonged impact on mental state. The current study has, for the first time, been able to lay a theoretical and methodological framework for future trials whereby the effectiveness of therapeutic interventions on health status can be assessed during an acute exacerbation of chronic obstructive pulmonary disease. Furthermore, the study shows that timing of health status determination post-exacerbation is very important, and that with progress in the management of chronic obstructive pulmonary disease, therapeutic interventions that speed health status recovery will become criteria in the treatment of the disease.

\section{ACKNOWLEDGEMENTS}

The authors would like to thank the principal investigators and study coordinators for their work in conducting the clinical trial.

\section{REFERENCES}

1 Bourbeau J, Julien M, Maltais F, et al. Reduction of hospital utilization in patients with chronic obstructive pulmonary disease: a disease-specific self-management intervention. Arch Intern Med 2003; 163: 585-591.

2 Collet JP, Shapiro P, Ernst P, Renzi T, Ducruet T, Robinson A. Effects of an immunostimulating agent on acute exacerbations and hospitalizations in patients with chronic obstructive pulmonary disease. The PARI-IS Study Steering Committee and Research Group. Prevention of acute respiratory infection by an immunostimulant. Am J Respir Crit Care Med 1997; 156: 1719-1724.

3 Connors AF Jr, Dawson NV, Thomas C, et al. Outcomes following acute exacerbation of severe chronic obstructive lung disease. The SUPPORT investigators (Study to Understand Prognoses and Preferences for Outcomes and Risks of Treatments). Am J Respir Crit Care Med 1996; 154: 959-967. 
4 Donaldson GC, Seemungal TA, Bhowmik A, Wedzicha JA. Relationship between exacerbation frequency and lung function decline in chronic obstructive pulmonary disease. Thorax 2002; 57: 847-852.

5 Kanner RE, Anthonisen NR, Connett JE. Lower respiratory illnesses promote FEV1 decline in current smokers but not ex-smokers with mild chronic obstructive pulmonary disease: results from the lung health study. Am J Respir Crit Care Med 2001; 164: 358-364.

6 Donaldson GC, Seemungal TA, Patel IS, et al. Airway and systemic inflammation and decline in lung function in patients with COPD. Chest 2005; 128: 1995-2004.

7 Miravitlles M, Ferrer M, Pont A, et al. Effect of exacerbations on quality of life in patients with chronic obstructive pulmonary disease: a 2 year follow up study. Thorax 2004; 59: 387-395.

8 Seemungal TA, Donaldson GC, Paul EA, Bestall JC, Jeffries DJ, Wedzicha JA. Effect of exacerbation on quality of life in patients with chronic obstructive pulmonary disease. Am J Respir Crit Care Med 1998; 157: 1418-1422.

9 Spencer S, Jones PW. Time course of recovery of health status following an infective exacerbation of chronic bronchitis. Thorax 2003; 58: 589-593.

10 Jones PW, Quirk FH, Baveystock CM, Littlejohns P. A selfcomplete measure of health status for chronic airflow limitation. The St. George's Respiratory Questionnaire. Am Rev Respir Dis 1992; 145: 1321-1327.

11 Jones PW, Bosh TK. Quality of life changes in COPD patients treated with salmeterol. Am J Respir Crit Care Med 1997; 155: 1283-1289.

12 Anie KA, Jones PW, Hilton SR, Anderson HR. A computerassisted telephone interview technique for assessment of asthma morbidity and drug use in adult asthma. J Clin Epidemiol 1996; 49: 653-656.

13 Rodriguez-Roisin R. Toward a consensus definition for COPD exacerbations. Chest 2000; 117, 5: Suppl. 2, 398S-401S.

14 Medical Research Council Committee on the Aetiology of Chronic Bronchitis. Standardized questionnaires on respiratory symptoms. BMJ 1960; 2: 1665.

15 EuroQol--a new facility for the measurement of healthrelated quality of life. The EuroQol Group. Health Policy 1990; 16: 199-208.

16 Garcia-Aymerich J, Monso E, Marrades RM, et al. Risk factors for hospitalization for a chronic obstructive pulmonary disease exacerbation. EFRAM study. Am J Respir Crit Care Med 2001; 164: 1002-1007.

17 Seemungal TA, Donaldson GC, Bhowmik A, Jeffries DJ, Wedzicha JA. Time course and recovery of exacerbations in patients with chronic obstructive pulmonary disease. Am J Respir Crit Care Med 2000; 161: 1608-1613.

18 BTS guidelines for the management of chronic obstructive pulmonary disease. The COPD Guidelines Group of the Standards of Care Committee of the BTS. Thorax 1997; 52: Suppl. 5, S1-28.

19 O'Donnell DE, Aaron S, Bourbeau J, et al. Canadian Thoracic Society recommendations for management of chronic obstructive pulmonary disease--2003. Can Respir J 2003; 10: Suppl. A, 11A-65A.

20 Barr RG, Bourbeau J, Camargo Jr CA, Ram FS. Tiotropium for stable chronic obstructive pulmonary disease: a metaanalysis. Thorax 2006; 61: 854-862.

21 Burge P. EUROSCOP, ISOLDE and the Copenhagen city lung study. Thorax 1999; 54: 287-288.

22 Calverley P, Pauwels R, Vestbo J, et al. Combined salmeterol and fluticasone in the treatment of chronic obstructive pulmonary disease: a randomised controlled trial. Lancet 2003; 361: 449-456.

23 Calverley P, Boonsawat W, Cseke Z, Zhong N, Peterson S, Olsson $\mathrm{H}$. Maintenance therapy with budesonide and formoterol in chronic obstructive pulmonary disease. Eur Respir J 2003; 22: 912-919.

24 Mahler DA, Wire P, Horstman D, et al. Effectiveness of fluticasone propionate and salmeterol combination delivered via the Diskus device in the treatment of chronic obstructive pulmonary disease. Am J Respir Crit Care Med 2002; 166: 1084-1091.

25 Szafranski W, Cukier A, Ramirez A, et al. Efficacy and safety of budesonide/formoterol in the management of chronic obstructive pulmonary disease. Eur Respir J 2003; 21: 74-81.

26 Wilkinson TM, Donaldson GC, Hurst JR, Seemungal TA, Wedzicha JA. Early therapy improves outcomes of exacerbations of chronic obstructive pulmonary disease. Am J Respir Crit Care Med 2004; 169: 1298-1303.

27 Sedeno MF, Nault D, Hamd D, Bourbeau J. A written action plan for early treatment of COPD exacerbations: An important component to the reduction of hospitalizations. Am J Respir Crit Care Med 2006; 3: A603. 